\title{
An Influence of the Estimated Glomerular Filtration Rate on Improvement in Metabolic Parameters by Sodium-Glucose Cotransporter 2 Inhibitors
}

\author{
Hisayuki Katsuyama ${ }^{\text {a }}$, Hidekatsu Yanai ${ }^{a, b}$
}

\section{To the Editor}

We systematically reviewed the anti-atherosclerotic effects beyond glucose lowering of sodium-glucose cotransporter 2 (SGLT2) inhibitors in patients with diabetes, and found that SGLT-2 inhibitors are proved to be significantly associated with weight loss and reduction of blood pressure, in addition to lowering plasma glucose, by a relatively large number of studies [1]. However, the studies investigating effects of SGLT2 inhibitors on visceral fat, insulin sensitivity, serum lipids, inflammation and adipocytokines were very limited [1]. Recently, we reported the effects of SGLT2 inhibitors on metabolic parameters in patients with type 2 diabetes [2]. Our study demonstrated that SGLT2 inhibitors significantly reduced $\mathrm{HbA} 1 \mathrm{c}$ and body weight and improved liver functions [2]. SGLT2 is expressed in the proximal tubule of kidney and mediates reabsorption of approximately $90 \%$ of the filtered glucose [3]. SGLT2 inhibitors block reabsorption of filtered glucose by inhibiting SGLT2, and promote the renal excretion of glucose [4]. Currently, several SGLT2 inhibitors with various degrees of selectivity toward SGLT2 versus SGLT1 selectivity are being tested in clinical trials [5]. I raised the issue of a significance of SGLT1 inhibition by SGLT2 inhibitors as my expert opinion [6]. Canagliflozin has a low potency to inhibit SGLT1 [7], which is highly distributed in intestine, and may improve postprandial hyperglycemia due to blocking intestinal absorption of glucose by inhibiting SGLT1. Tofogliflozin was identified as a potent and highly selective SGLT2 inhibitor [8]. I found a significant difference in hypoglycemic effect between canagliflozin and tofogliflozin [6], and suggested that a significance of SGLT1 inhibition and selectivity for SGLT2 are the issues that cannot be ignored. I also recommended that we should study how SGLT1 inhibition and selectivity for

Manuscript accepted for publication March 18, 2016

a Department of Internal Medicine, National Center for Global Health and Medicine Kohnodai Hospital, Chiba, Japan

${ }^{\mathrm{b}}$ Corresponding Author: Hidekatsu Yanai, Department of Internal Medicine, National Center for Global Health and Medicine Kohnodai Hospital, 1-7-1 Kohnodai, Ichikawa, Chiba 272-0034, Japan.

Email: dyanai@hospk.ncgm.go.jp

doi: http://dx.doi.org/10.14740/jocmr2547w
SGLT2 have an influence on glucose control, and we should elucidate whether such influence is different depending on the patients or not. As the key to resolve these issues, a different distribution between SGLT1 (intestine) and SGLT2 (kidney) came to my mind. SGLT2 inhibitors with potency to inhibit SGLT1 may be more effective to lower glucose as compared with highly selective SGLT2 inhibitors, in patients with renal insufficiency.

To understand an influence of the estimated glomerular filtration rate (eGFR) on improvement in metabolic parameters by SGLT2 inhibitors, we sub-analyzed our previous study [2]. We studied 48 patients with type 2 diabetes. Baseline characteristics of subjects who had taken SGLT2 inhibitors and SGLT2 inhibitors prescribed to subjects at baseline are shown in Tables 1 and 2, respectively. The mean value of eGFR in subjects was $93.1 \mathrm{~mL} / \mathrm{min} / 1.73 \mathrm{~m}^{2}$. We divided patients into the high eGFR group (mean $\pm \mathrm{SD}$ of eGFR, $117 \pm 36 \mathrm{~mL} /$ $\left.\mathrm{min} / 1.73 \mathrm{~m}^{2} ; \mathrm{n}=22\right)$ and the low eGFR group $(72 \pm 14 \mathrm{~mL} /$ $\left.\min / 1.73 \mathrm{~m}^{2} ; \mathrm{n}=26\right)$ by the mean value of eGFR. The changes in $\mathrm{HbAlc}$, body weight and alanine aminotransferase (ALT) at 1, 2, 3 and 6 months after the start of SGLT2 inhibitors are shown in Figure 1. HbA1c significantly decreased in the high eGFR group as compared with the low eGFR group at 1,2, 3 and 6 months after the SGLT2 inhibitors started. The changes in HbAlc at 2 months $(\mathrm{r}=-0.361, \mathrm{P}=0.059$, by the Pearson's

Table 1. Baseline Characteristics of Subjects Who Had Taken SGLT2 Inhibitors $(n=48)$

\begin{tabular}{ll}
\hline Baseline characteristics & \\
\hline Age (years) & $48 \pm 11$ \\
Sex (male/female) & $16 / 32$ \\
Body height $(\mathrm{cm})$ & $161 \pm 8$ \\
Body weight $(\mathrm{kg})$ & $85.2 \pm 17.9$ \\
Body mass index (BMI) $\left(\mathrm{kg} / \mathrm{m}^{2}\right)$ & $32.2 \pm 6.0$ \\
Systolic blood pressure $(\mathrm{mm} \mathrm{Hg})$ & $130 \pm 15$ \\
Diastolic blood pressure $(\mathrm{mm} \mathrm{Hg})$ & $80 \pm 11$ \\
Alanine aminotransferase $(\mathrm{IU} / \mathrm{L})$ & $52 \pm 41$ \\
eGFR (mL/min/1.73 $\left.\mathrm{m}^{2}\right)$ & $93 \pm 35$ \\
Plasma glucose (mg/dL) & $178 \pm 75$ \\
HbA1c $(\%)$ & $8.9 \pm 2.0$ \\
\hline
\end{tabular}


Table 2. SGLT2 Inhibitors Prescribed to Subjects at Baseline $(n=48)$

\begin{tabular}{ll}
\hline SGLT2 inhibitors & \\
\hline Dapagliflozin $5 \mathrm{mg}$ & $18(38 \%)$ \\
\hline Ipragliflozin $25 \mathrm{mg}$ & $3(6 \%)$ \\
Ipragliflozin $50 \mathrm{mg}$ & $9(19 \%)$ \\
Tofogliflozin $20 \mathrm{mg}$ & $6(13 \%)$ \\
Luseogliflozin $2.5 \mathrm{mg}$ & $10(46 \%)$ \\
Canagliflozin $100 \mathrm{mg}$ & $1(2 \%)$ \\
Empagliflozin $10 \mathrm{mg}$ & $1(2 \%)$ \\
\hline
\end{tabular}

correlation) and 3 months $(\mathrm{r}=-0.349, \mathrm{P}=0.063)$ after the start of SGLT2 inhibitors tended to be negatively correlated with the baseline eGFR value. There were no significant differences in the changes in body weight between the high eGFR group and the low eGFR group, and the changes in body weight were not correlated with the baseline eGFR value at any points of time. There were no significant differences in the changes in ALT between the high eGFR group and the low eGFR group at any points of time. However, the change in ALT at 2 months after the start of the SGLT2 inhibitors tended to be negatively correlated with the baseline eGFR value $(\mathrm{r}=-0.329, \mathrm{P}=$ $0.066)$, and the changes in ALT at 3 months $(\mathrm{r}=-0.554, \mathrm{P}=$ $0.001)$ and 6 months $(\mathrm{r}=-0.466, \mathrm{P}=0.025)$ were significantly and negatively correlated with the baseline eGFR value.

The present study demonstrated that eGFR has an influence on the improvement in $\mathrm{HbAlc}$ and liver function (ALT) by SGLT2 inhibitors. However, eGFR did not show an influence on the change in body weight by SGLT2 inhibitors. Although the underlying mechanism for such difference remains unknown, our study may facilitate further studies to elucidate a significance of SGLT1 inhibition by SGLT2 inhibitors.

\section{References}

1. Yanai H, Katsuyama H, Hamasaki H, Adachi H, Moriyama S, Yoshikawa R, Sako A. Sodium-Glucose Cotransporter 2 Inhibitors: Possible Anti-Atherosclerotic Effects Beyond Glucose Lowering. J Clin Med Res. 2016;8(1):1014.

2. Katsuyama H, Hamasaki H, Adachi H, Moriyama S, Kawaguchi A, Sako A, Mishima S, et al. Effects of Sodi-
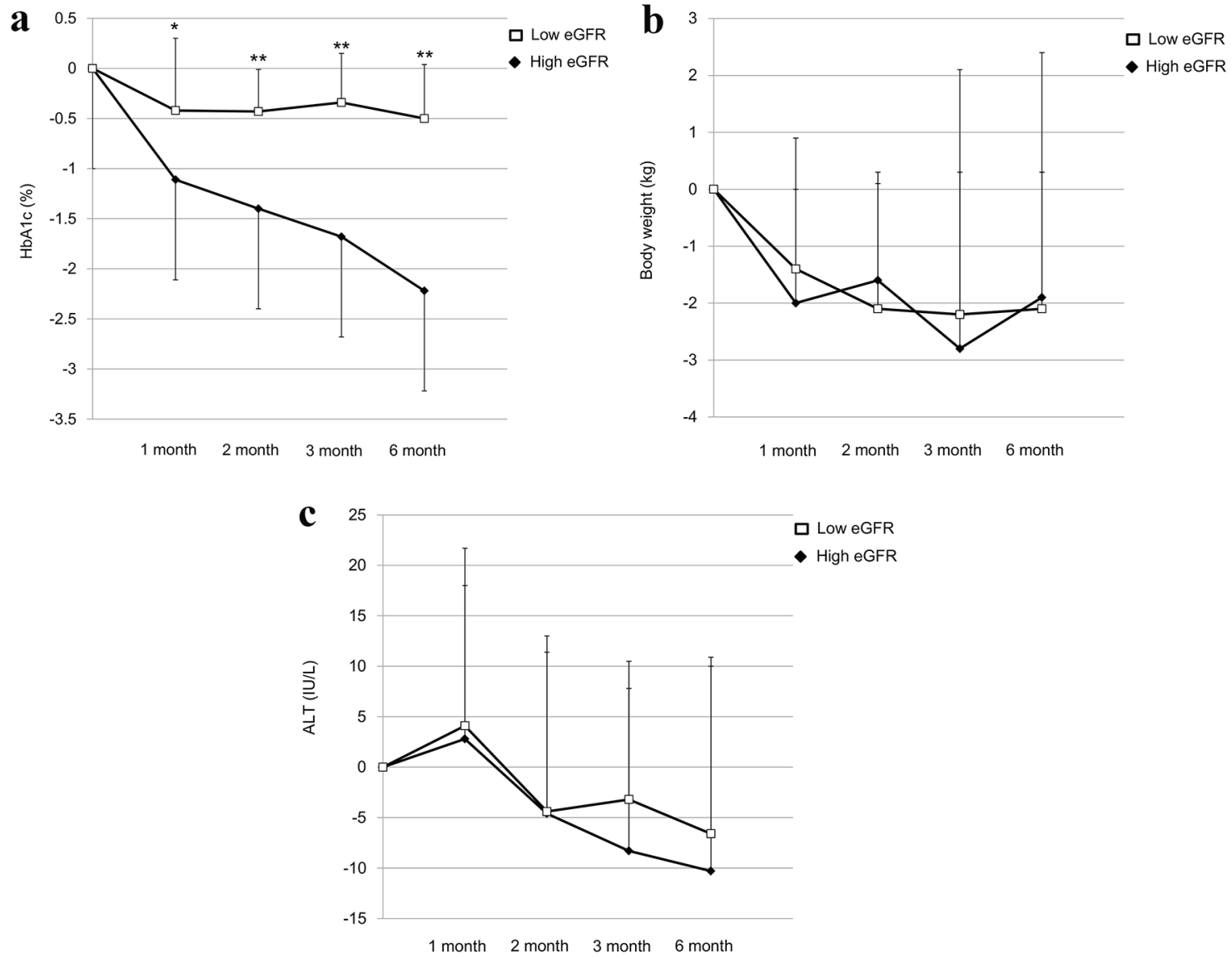

Figure 1. The changes in $\operatorname{HbA1c}(a)$, body weight (b) and ALT (c) at 1, 2, 3 and 6 months after the start of SGLT2 inhibitors in the low eGFR group and the high eGFR group. ${ }^{*} \mathrm{P}<0.05$ and ${ }^{* *} \mathrm{P}<0.01$ vs. the high eGFR group by the paired Student's $t$-test, respectively. ALT: alanine aminotransferase; eGFR: estimated glomerular filtration rate; SGLT2: sodium-glucose cotransporter 2. 
um-Glucose Cotransporter 2 Inhibitors on Metabolic Parameters in Patients With Type 2 Diabetes: A Chart-Based Analysis. J Clin Med Res. 2016;8(3):237-243.

3. Vallon V, Platt KA, Cunard R, Schroth J, Whaley J, Thomson SC, Koepsell H, et al. SGLT2 mediates glucose reabsorption in the early proximal tubule. J Am Soc Nephrol. 2011;22(1):104-112.

4. Jabbour SA, Goldstein BJ. Sodium glucose co-transporter 2 inhibitors: blocking renal tubular reabsorption of glucose to improve glycaemic control in patients with diabetes. Int J Clin Pract. 2008;62(8):1279-1284.

5. Musso G, Gambino R, Cassader M, Pagano G. A novel approach to control hyperglycemia in type 2 diabetes: sodium glucose co-transport (SGLT) inhibitors: systematic review and meta-analysis of randomized trials. Ann Med. 2012;44(4):375-393.

6. Yanai H. A Significance of Sodium-Glucose Cotransporter 1 (SGLT1) Inhibition by SGLT2 Inhibitors. Int J Diabetes Clin Diagn. 2015;1:102.

7. Scheen AJ, Paquot N. Metabolic effects of SGLT-2 inhibitors beyond increased glucosuria: A review of the clinical evidence. Diabetes Metab. 2014;40(6 Suppl 1):S4-S11.

8. Ohtake Y, Sato T, Kobayashi T, Nishimoto M, Taka N, Takano K, Yamamoto K, et al. Discovery of tofogliflozin, a novel $\mathrm{C}$-arylglucoside with an $\mathrm{O}$-spiroketal ring system, as a highly selective sodium glucose cotransporter 2 (SGLT2) inhibitor for the treatment of type 2 diabetes. J Med Chem. 2012;55(17):7828-7840. 